\title{
A Lempel-Ziv Like Approach for Signal Classification*
}

\author{
J. MONTALVÃO ${ }^{1 * *}$ and J. CANUTO ${ }^{2}$
}

Received on December 26, 2011 / Accepted on July 4, 2014

\begin{abstract}
In this paper, the seminal method proposed by Abraham Lempel and Jacob Ziv, aimed at the complexity analysis of sequences of symbols, was modified to compare similarities between two sequences. This modification allowed the creation of a new criterion which can replace likelihood in some pattern recognition applications. Moreover, to allow for analysis and comparison of multivariate continuously valued patterns, we also present a simple adaptation of the Lempel-Ziv's method to time-sampled signals. To illustrate the usefulness of these proposed tools, two sets of experimental results are presented, namely: one on speaker identity verification (biometrics) and another on healthcare signal detection. Both experiments yield promising performances. Moreover, as compared to a conventional pattern recognition method, the new approach provided better performances in terms of Equal Error Ratio in speaker verification experiments.
\end{abstract}

Keywords: signal analysis, no a priori, Lempel-Ziv complexity.

\section{INTRODUCTION}

In 1976, A. Lempel and J. Ziv [1] proposed an approach for complexity analysis of symbol sequences. An important aspect of their approach is the lack of a priori with regard to the source of symbols, which clearly contrasts with the measurement of (source) Shannon entropy [2]. Though they are conceptually different measures, it was shown that [3], under ergodicity conditions, Lempel-Ziv's complexity of increasingly long symbol sequences converges almost surely to the Shannon entropy of the source from which symbols are drawn.

Lempel-Ziv's (LZ) approach, latterly simplified for practical reasons, became widely known as the compression algorithm behind many computer programs for file compression - the "zip-like" programs. We should probably credit its success to its universality, in other words, to its lack of a priori. Nevertheless, it should be also highlighted that zip-like programs are just the "tip of the iceberg," for compression is just a single offspring of the elegant theory presented in [1].

\footnotetext{
*This work was granted by the Conselho Nacional de Desenvolvimento Científico e Tecnológico (CNPq).

**Corresponding author: Jugurta Montalvão.

${ }^{1}$ Departamento de Engenharia Elétrica, UFS - Universidade Federal de Sergipe, 49000-000 São Cristóvão, SE, Brazil.

E-mail: jmontalvao@ufs.br

2 Département EPH, Télécom SudParis, 9 rue Charles Fourier, 91011 EVRY, France.

E-mail: Janio.Canuto@it-sudparis.eu
} 
Indeed, compression is a consequence of redundancy removal, and "zip-like" programs aim at finding redundancies in streams of symbols, regardless of what they represent (e.g. texts, audio, video). These redundancies may even be gathered in a so-called "dictionary", whose content corresponds to unique non-overlapping segments of the analyzed sequence of symbols. We highlight though that the idea of an explicit dictionary does not take part in the original analysis method proposed in [1]. Therefore, the dictionary definition in this paper is arbitrary.

The original method by Abraham Lempel and Jacob Ziv was aimed at symbolic sequence analysis, but it may be adapted to work with sequences of numbers as well (e.g. sampled signals). For instance, this adaptation can be done rather straightforwardly through simple quantization of the signal, thus mapping it back into a sequence of labels (one label per quantization level). In all cases, redundancy analysis allows for: compression, segmentation, pattern recognition and/or prediction.

Signal comparison through LZ approach is not new, though not too current in the pattern recognition scenario. One rather isolated example (to the extent of the authors' knowledge), published in 1995, is the work by P. Johansen [4], in which handwritten signature authenticity is verified (behavioural biometrics). Indeed, the asymmetric measure defined in Section 4 is closely related to the ideas presented in [4]. In [5], it was clearly shown, through numeric examples, that LZ complexity may replace the Lyapunov exponent as a more precise measure of order/disorder, in spatiotemporal pattern analysis. Besides, in [6] the Lempel-Ziv-Welch algorithm was successfully applied to texture (image) classification.

In spite of the above mentioned examples, we also observe that LZ based signal analysis is more common in specific research domains, such as biomedical signals $[8,7,9,10]$, possibly due to the nice properties of the LZ-based entropy estimators, as compared to the plug-in method $[11,7]$. Moreover, because LZ algorithms only process sequences of symbols, thresholding and labeling are frequently applied to real-valued signals prior to LZ analysis. Indeed, in most cases, a single threshold is used to generate two-symbol sequences. For instance, in [4] binary pixel attributes were used (black or white pixels), and in [5] binary sequences were obtained through the use of two thresholding methods: an adaptive single threshold for series from a pseudorandom-number-generator, and a fixed threshold, at 0.5 , for series generated by logistic map difference equations. In [8], a study of the influence of the thresholding method on LZ complexity measure is presented, considering more than one threshold (more than two symbols in resulting sequences). Unfortunately, that work does not take into account multivariate signals, whose quantization is a more complex matter.

Similarly, in [12], nine groups of signals were analysed with a pool of complexity measures, including Lempel-Ziv's one. Again, the simplest procedure to express multivariate time series of dynamical data as a symbolic sequence was used there. This procedure was the calculation of Euclidean distances between consecutive points, followed by the comparison of these distances to their median, thus yielding two-symbol sequences.

In this paper, we address multivariate signal comparison with a LZ-like method. To properly explain our approach, we first present the LZ method in Section 2. Then, in Section 3, we 
describe a new method which allows for LZ analysis of continuous multivariate sampled signals. In Section 4, a new similarity measure between signals is proposed, inspired by the complexity measure defined in [1]. Moreover, in Section 5, it is shown how to use this new measure as an alternative to likelihood based criteria. Also in that Section 5, we gather some experimental results with and without vector quantization of signals from two public databases. These results are analysed and conclusions are presented in Section 6.

\section{THE LEMPEL-ZIV'S METHOD}

Let $s_{1}^{n}$ be a sequence of $n$ symbols drawn from a finite alphabet, $\mathcal{A}$, Lempel-Ziv's complexity analysis is based on the parsing of $s_{1}^{n}$ into a minimum number of unique (with one possible exception) subsequences of symbols. Though the idea of a dictionary of subsequences is not presented in the seminal paper published in 1976 [1], we believe that it is a powerful point of view for pattern recognition. Therefore, we define a growing dictionary of subsequences $s_{i}^{j}$, where $s_{i}^{j}$ stands for a substring formed by symbols from position $i$ to position $j(i \leq j \leq n)$. Thus, Lempel-Ziv's algorithm can be summarized as follows:

1. Set $k=1, L=1$ and start with a single element in the dictionary, $\left\{s_{1}^{1}\right\}$. Set a pointer to the first symbol of the sequence, $p_{k}=1$.

2. Increase $L$ by one, $L \leftarrow L+1$. If $p_{k}+L-1$ equals $n$, then take the new subsequence of length $L, s_{p_{k}}^{p_{k}+L-1}$, as the last parsed segment and stop the algorithm, otherwise...

3. Compare $s_{p_{k}}^{p_{k}+L-1}$ (a substring of length $L$ ) to every subsequence of the same length in the past $^{3}, s_{1}^{p_{k}+L-2}$.

4. If this search fails:

- The subsequence $s_{p_{k}}^{p_{k}+L-1}$ is given as a new dictionary entry;

- $p_{k}$ is set to the position of the next symbol, $p_{k} \leftarrow p_{k}+L$;

- $k$ is increased by one, $k \leftarrow k+1$;

- $L$ is set to zero, $L \leftarrow 0$, and;

- the algorithm flow moves back to step (2).

Otherwise, go to step 2.

The number of parsed substrings through this process, represented by $C\left(s_{1}^{n}\right)$, was proposed by Lempel and Ziv as a complexity measure.

\footnotetext{
${ }^{3}$ An alternative method was proposed in 1978 [13] to alleviate the computational burden, in which new subsequences are compared to subsequences of the same length in the dictionary.
} 


\section{PARSING TIME-SAMPLED SIGNALS WITHOUT VECTOR QUANTIZATION}

To allow for signal replacing sequences of non-numerical symbols, we stretch a little bit our notation and let $s_{1}^{n}$ also stand for a sequence of $n$ signal samples, taken at regular time intervals. Thus, $s_{i}^{j}$ stands for a sequence of $j-i+1$ signal samples drawn from a possibly infinite set (e.g. the Real set). Note that regular time-sampling is assumed, but quantization of samples is not mandatory. Nevertheless, for the convenience of computational experiments in this paper, we use quantized signals instead of continuously valued samples.

Because the domain of $s_{i}$ may be continuous, if we apply the Lempel-Ziv algorithm to it, we may end up with an infinite dictionary of single samples (because every new sample may be unique!). A simple way to cope with this problem is to quantize samples. For instance, a 'wav' file, with 16 bits per sample (65536 quantization levels), can be partially compressed with the LZ algorithm, although with a poor performance, less than $60 \%$ (compressed size/original size $\times 100$ ), on average. Roughly speaking, both the choice of the number of quantization levels and the quantization scheme (linear/nonlinear) clearly influence the compression result.

By contrast, we are not bound to quantization to allow for comparisons between signal segments. By regarding a sequence of samples, $s_{i}^{i+L-1}$, as an instance of a discrete-time stochastic process [3] of length $L$, we may use pattern recognition methods[14] to decide whether an observed instance is "new" or if it already appeared in the signal past. If it is new, then it is given as a dictionary entry, thus forming a dictionary which is a set of signal segments, as illustrated in Figure 1.

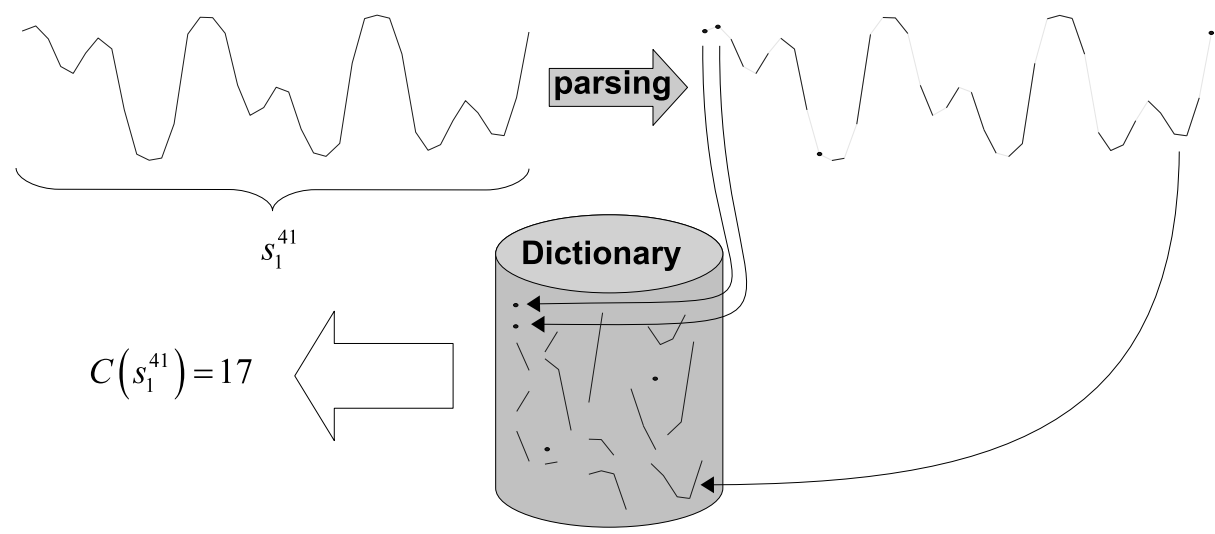

Figure 1: An illustration of Lempel-Ziv's complexity analysis applied to continuously valued (time sampled) signals.

A straightforward way to adapt the LZ algorithm to continuously valued signals is the replacement of step (3), in Section 2 with:

Modified step (3): Compare $s_{p_{k}}^{p_{k}+L-1}$ (now a signal segment of length $L$ ) to every signal segment of the same length in the "past," $s_{1}^{p_{k}+L-2}$. It is important to note that every single $s_{i}$ may now 
be a pattern laying in $\mathcal{R}^{D}$, as far as the signal can be an instance of a multivariate random process of dimension $D$.

In typical pattern recognition approaches, this comparison depends on a random vector model, i.e. multivariate probability density functions (pdf). In order to keep the pivotal idea in the LZ approach, we also should estimate this pdf explicitly or implicitly through a method without $a$ priori, which leads us to nonparametric methods, such as the Parzen or the K-Nearest Neighbours (KNN) [14]. Unfortunately, both methods involve a training step which may be too computationally demanding. Indeed, in this case, we should estimate one pdf per value of $L$, to be re-estimated every new comparison because the training set - past signal - changes all the time.

As a strong simplification, we assume that both non-parametric methods, Parzen and KNN, ultimately take decisions by comparing distances between a new signal segment and reference segments of the same length. These distances are then compared to a threshold. Thus, our simplified approach for signal comparison, in Modified step (3), is:

- Arbitrarily set an Innovation Threshold, $T_{I}$.

- Copy $s_{p_{k}}^{p_{k}+L-1}$ to a matrix $S_{i, j}^{(0)}$, where $1 \leq j \leq L$ and $1 \leq i \leq D$.

- Copy the $r^{t h}$ signal segments of length $L$ from $s_{1}^{p_{k}+L-2}\left(1 \leq r \leq p_{k}+L-1\right)$ to matrix $S_{i, j}^{(r)}$.

- Compute $p_{k}+L-1$ mean squared deviation as: $d^{(r)}=\frac{1}{D+L} \sum_{i=1}^{D} \sum_{j=1}^{L}\left(S_{i, j}^{(0)}-S_{i, j}^{(r)}\right)^{2}$.

- If at least one $d^{(r)}$ is less than $T_{I}$, then $s_{p_{k}}^{p_{k}+L-1}$ is not new (for a noisy copy of it was found in the past), and the search succeeds, otherwise it fails, and the signal segment is considered as an innovation.

\section{A NEW SIMILARITY MEASURE FOR PATTERN RECOGNITION}

Pattern classification is usually based on likelihood from probabilistic models, or distances between patterns which can loosely be regarded as simplifications of likelihood based criteria. In this paper, we claim that compression rate may play a role similar to likelihood. Considering a lossless compression scheme, the higher the compression rate of a given signal, the greater is the number of long segments of samples that can be found more than once in the whole signal. In order to use compression rate to compare two signals, we modify the LZ algorithm to consider two series as inputs, where one of them just plays the role of "past signal", and the other is parsed.

Let $x_{1}^{m}$ and $y_{1}^{n}$ be two sequences of $m$ and $n$ symbols, both drawn from a finite alphabet, $\mathcal{A}$. Alternatively, $x_{1}^{m}$ and $y_{1}^{n}$ may be two sampled signals, both with samples lying in $\mathcal{R}^{D}$. The Modified Lempel-Ziv (MLZ) parsing procedure starts by searching for $y_{1}^{p_{1}}$ (initially $p_{1}=1$ ) inside $x_{1}^{m}$. If this search succeeds, $p_{1}$ is replaced with $p_{1}+1$ and a new search is done, otherwise the pointer $p_{1}$ is registered, and a new pointer $p_{2} \leftarrow p_{1}+1$ is created, and the search in $x_{1}^{m}$ for a subsequence equal/similar to $y_{p_{1}+1}^{p_{2}}$ is done; and so on. The algorithm stops when the end of $y_{1}^{n}$ 
is reached. The number of segments into which $y_{1}^{n}$ is parsed is denoted by $C_{M L Z}\left(y_{1}^{n} ; x_{1}^{m}\right)$, thus indicating that $x_{1}^{m}$ plays the role of a parameter of $C_{M L Z}$.

Illustration: If $x_{1}^{6}=a b b a a b$ and $y_{1}^{9}=a b a b b a a b b$, the modified parsing process produces: $a b a \cdot b b a a b b$. By denoting it as a function of $y$, parametrized by $x$, we write: $C_{M L Z}(y ; x)=2$.

It is worth noting that measure $C_{M L Z}\left(y_{1}^{n} ; x_{1}^{m}\right)$ depends on $m$, the length of "parameter" $x_{1}^{m}$. Parameter $x_{1}^{m}$ can be regarded as a memory of subsequences of symbols/samples, and the longer such memory is, the more likely we are to find longer segments of $y$ in $x$, even if $y$ and $x$ are generated by statistically independent sources. To properly compensate for this dependency of the proposed measure, $C_{M L Z}$ on $m$, we make an appeal to an important statement in [1], according to which the maximum complexity measure of a sequence $x_{1}^{m}$, produced by an ergodic $\alpha$-symbol source ( $\alpha$ is the cardinality of $\mathcal{A}$ ), is $m / \log _{\alpha}(m)$. This upper bound gives a clue concerning the averaged length of dictionary items (sub-sequences of symbols) produced by LZ processing of $x_{1}^{m}$. That is to say that $m / \log _{\alpha}(m)$ estimates the number of segments into which $x_{1}^{m}$ is parsed, by assuming an averaged segment length of $\log _{\alpha}(m)$.

As a result, we claim that the averaged length of segments of $y_{1}^{n}$ found in $x_{1}^{m}$, given by

$$
\frac{n}{C_{M L Z}\left(y_{1}^{n} ; x_{1}^{m}\right)},
$$

is a measure of similarity between the two sequences, but it depends on $m$. In order to make this measure less sensitive to the length of $x_{1}^{m}$, we divide it by $\log (m)$ (the logarithm base is not relevant, because its change corresponds to multiplying the measure by a constant scale factor). Accordingly, we propose a new similarity criterion between sequences of symbols, given by:

$$
S\left(y_{1}^{n} ; x_{1}^{m}\right)=\frac{n}{C_{M L Z}\left(y_{1}^{n} ; x_{1}^{m}\right) \log (m)}
$$

and because this measure is not symmetric, i.e. $S\left(y_{1}^{n} ; x_{1}^{m}\right) \neq S\left(x_{1}^{m} ; y_{1}^{n}\right)$, we finally propose a symmetric criterion, given by:

$$
J\left(y_{1}^{n}, x_{1}^{m}\right)=(1 / 2)\left(S\left(y_{1}^{n} ; x_{1}^{m}\right)+S\left(x_{1}^{m} ; y_{1}^{n}\right)\right) ;
$$

\section{EXPERIMENTAL RESULTS}

A straightforward approach to Lempel-Ziv analysis of sequences of numeric patterns is to vector quantize it and to perform analysis on the resulting sequence of labels (e.g. prototype labels). Though it is indeed a straightforward solution, it raises some nontrivial questions, such as the influence of number of prototypes and quantization strategy on the analysis result. By contrast, the new algorithm proposed in Section 3 allow for LZ analysis of multivariate signals without quantization. Nonetheless, it also raises difficult questions concerning the choice of the threshold $T_{I}$. In this paper, we do not discuss such difficult matters, which are postponed to future works. Instead, we empirically study the influence of both the number of prototypes, $K$ (approach with quantization), and the threshold, $T_{I}$ (approach without signal quantization), on signal classification performances, through error ratio measures. Moreover, we also gather some empirical evidences concerning the usefulness of the criterion defined in Eq. 4.2 in pattern recognition. 
Thus, in this Section, we present results from two sets of experiments. First in biometric speaker verification, and secondly in healthcare signal detection. Both experiments were done with publicly available databases, so that it can be easily reproduced by interested readers.

\subsection{Experiment with speaker recognition (Biometrics)}

The speaker verification experiment was done with a publicly available database (The BioChaves database, available at http://www.biochaves.com/). Speech samples in this database correspond to signals recorded during the uttering of a single set of four words in Portuguese - "chocolate, zebra, banana, táxi." — identically spelled in English, apart from the spelling of "táxi”. The duration of each utterance is about $3 s$ and each subject uttered this set of words 10 times, 5 (five) samples during a first session, and 5 more samples during a second session, about a month later, using a conventional headset (electret microphone plus headphone). Speech signals were thus digitalized and recorded with 16 bits per sample, at 22050 samples per second. All recordings were done under low background noise, though in an uncontrolled environment. Ten subjects took part in the experiment.

In terms of biometric verification, it is a small database. However, since our goal is to compare pattern recognition approaches based on the very same MFCC extracted from recorded utterances, this database provide us with a total of 1375 distinct pair-wise comparisons between utterances from different sessions (1125 from different speakers and 250 from the same one) which is enough to show a clear and consistent difference between approach performances.

By doing pair-wise comparisons we simulate a verification protocol with 1 utterance per enrollment, and 1 per interrogation. Thus, given the too short duration of each utterance for biometric purposes - only $3 \mathrm{~s}$-, we should expect high error ratios, as compared to typical biometric experiments in literature. But it does not matter in this work, as we are interested in the comparative performance of the tested algorithms.

Therefore, in each experiment, every single utterance from one session (half database) is taken once as a prototype (enrollment) and then cross-compared to every other utterance from the other session. Whenever both compared utterances came from the same person, the resulting score is labeled with a ' $\mathrm{T}$ ' (true claimed identity), otherwise, it is labeled with an ' $\mathrm{F}$ '. As a result, each algorithm provides 250 scores labeled ' $T$ ', and 1125 labeled ' $F$ '. Then we adjust a decision threshold to minimize the Equal Error Rate (EER) - i.e. the operational point where false alarm rate (FAR) equals false rejection rate (FRR) — for each set of scores, for each algorithm.

All pattern recognition algorithms used here use 19 Mel-Frequency Cepstral Coefficients (MFCC) per time frame of $25 \mathrm{~ms}$, with overlapping between frames of $82 \%$ (advance of $4.5 \mathrm{~ms}$ per frame). Given all overlapping short frames of signal, in order to discard silent frames (or frames with too low acoustic energy), we first estimate the signal power in each frame, and then we set an adaptive energy threshold between the the minimum and the averaged power per frame. All remaining short-signal frames are then frequency analysed, yielding a matrix, $X_{i, j}$, of cepstral coefficients per recorded utterance, where $i,(1 \leq i \leq 19)$ stands for a coefficient index, and $j(j=1,2, \ldots)$ is a short-time frame counter. 
As for the vector quantization strategy, an important aspect of it is that matrices of patterns from two signals under comparison must share the same feature space, as well as the same set of prototypes. To induce it, being $X$ and $Y$ MFCC matrices extracted from two utterances (speech signals) to be compared, respectively, we first concatenate $X$ and $Y$ in a single bigger matrix $Z=\left[\begin{array}{ll}X & Y\end{array}\right]$, and then we find $K$ prototypes in 19-Dimensional feature space (for simplicity, we use standard K-Means algorithm [14]). Finally, each column of $X$ and $Y$ is labelled with the closest prototype index, thus yielding two sequences of labels, $x$ and $y$, respectively.

In our experiment with the BioChaves database, and 19-MFCC coefficients, we empirically found that the $K$ (number of prototypes) that minimizes the EER is $K=30$. By using the similarity measure defined in Eq. 4.2 to compare several instances of independently quantizedlabelled utterances, we obtained an averaged $E E R=7.6 \%$, with an $95 \%$ confidence interval of approximately $\pm 0.2 \%$. Note that, due to the random initialization of the K-Means algorithm, scores from a given pair of signals can be regarded as instances of a random variable.

This result, still with quantization-labelling of patterns, was sensibly improved with the averaging of 3 instances of corresponding values of $J(x, y)$. More precisely, for each pair of utterances, we performed 3 independent clustering and quantization of patterns, yielding three instances of $x$ and $y$, say $x^{(1)}, x^{(2)}, x^{(3)}, y^{(1)}, y^{(2)}$ and $y^{(3)}$. Then three corresponding scores were computed, namely $J\left(x^{(1)}, y^{(1)}\right), J\left(x^{(2)}, y^{(2)}\right)$ and $J\left(x^{(3)}, y^{(3)}\right)$. Finally, the mean of these three scores (for each pair of signals) was taken as final score.

This idea is inspired by the clustering ensemble strategy [15], where the agreement/disagreement between independent clusterings is used to stabilize and improve cluster analysis. Still according to the theory of clustering ensembles, diversity of partitions is beneficial. Here, we induce diversity by using three different number of prototypes per final score, $K_{1}, K_{2}$ and $K_{3}$, as indicated in in Table 1, where thresholds were optimised to minimize the EER.

Table 1: EER found with the LZ analysis with quantization - speaker recognition experiment.

\begin{tabular}{|c|c|c|}
\hline$K_{1}, K_{2}, K_{3}$ & EER $(\%) \pm 0.2$ & Score threshold \\
\hline $14,15,16$ & 6.8 & 0.24 \\
\hline $19,20,21$ & 5.7 & 0.23 \\
\hline $24,25,26$ & 5.6 & 0.20 \\
\hline $29,30,31$ & 5.3 & 0.18 \\
\hline $34,35,36$ & 6.8 & 0.17 \\
\hline
\end{tabular}

On the other hand, by applying the quantization-less algorithm described in Section 3 to the very same pairs of signals, and by comparing them with the same similarity measure defined in Eq. 4.2, we obtained the results presented in Table 2. Note that, in this case, we must carefully tune $T_{I}$, the innovation threshold, instead of $K$. Moreover, unlike the quantization based approach, scores from this method, for a given pair of signals, are no longer random. 
Though the best result in Table 2 was obtained with $T_{I}=0.3$, by finely tuning $T_{I}$ to 0.27 , a slightly better result was obtained: $E E R=5.1 \%$. By contrast, in [16], with the very same database, set of features (conventional MFCC) and biometric protocol (one utterance from first Session per enrollment, versus one utterance from second Session per interrogation), the minimum EER obtained with a classic pattern recognition approach was $\approx 7.5 \%$.

Table 2: EER found with the LZ analysis without quantization - speaker recognition experiment.

\begin{tabular}{|c|c|c|}
\hline Innov. threshold & EER (\%) & Score threshold \\
\hline 0.2 & 6.2 & 0.13 \\
\hline 0.3 & 5.2 & 0.15 \\
\hline 0.4 & 6.7 & 0.21 \\
\hline 0.5 & 7.4 & 0.29 \\
\hline 0.7 & 9.7 & 0.76 \\
\hline
\end{tabular}

Concerning the computational burden of both methods, any non-naive implementation of the MLZ Algorithm for comparing a reference string $x_{1}^{m}$ and a test string $y_{1}^{n}$, in the worst scenario where $x_{1}^{m}$ and $y_{1}^{n}$ don't have any common element, only requires $n m$ comparisons. By contrast, the K-NN algorithm needs the computation of all the distances between the $m$ reference points and the $n$ test points, in $d$-dimensional space (in this case $d=19$ ). This computation requires the execution of $d m n$ subtractions and power (multiplication) operations. The squared root operation is not necessary since it does not affect the sorting of such values. After the computation of the $m n$ distances, it is necessary to sort the $n$ groups of $m$ distances each. Since an efficient sorting algorithm for $m$ values (e.g. quicksort) has time complexity of $O(m \log (m))$, for the $n$ groups we have $O(m n \log (m))$. Therefore, the LZM is for the worst case $O(m n)$ (being usually much lower) whereas a classical approach with similar performance results needs two steps, the first one is $O(d m n)$ and the second one $O(m n \log (m))$.

\subsection{Experiment with accelerometer signals (Healthcare)}

The second set of experiments reported here is concerned with remote healthcare. More precisely, we analyze signals from an accelerometer attached to a subject under surveillance. These accelerometer signals are publicly available at the UCI repository [17] and was used in [18]. In our experiments, we use only signals from the belt sensor, as if it was registered by a single smartphone carried by the subject under medical surveillance. Moreover, we only gather three subsets of signals, corresponding to the following classes: (i) 'patient falling': 5 signals; (ii) 'patient lying': 5 signals; (iii) 'patient walking': 5 signals.

Each recorded signal corresponds to a set of 3-D vectors of regularly sampled measures in 3 orthogonal directions. More details concerning signal description and acquisition can be found in $[17,18]$. Here, unlike [18], we do not use context-dependent reasoning, but a much simpler approach based on direct signal comparisons to explore LZ capabilities on signal detection. 
Accordingly, the only signal processing technique applied to these multivariate signals is normalization of signal power for each channel (i.e. each orthogonal direction).

The experiment is carried out as follows: a single data file, out of 15, is taken as a reference. For instance, lets assume that a signal from 'subject falling' is arbitrarily taken as a single reference for 'falling' event. Then a second signal is randomly taken, playing the role of an online recording during actual patient monitoring. These two signals are compared, providing a score.

A detection threshold is adjusted to the point where false negative and false positive rates are the same (EER), and if a score from two signals from the same class is under this threshold, a 'false negative' event is computed. On the other hand, if a score from two signals from different classes lies above it, a 'false positive' is computed. In this case, both approaches (i.e. with and without quantization) yielded the same stable result: only 4 false negatives, all in class 'walking'.

As a matter of fact, the very same 4 recordings from class 'walking' were scored below threshold in both approaches, as illustrated in Figure 2, from one single but representative run of the method with quantization. Moreover, we consider this result as a stable one, because it remains unchanged for a wide range of parameter values, namely: (i) Quantization with a single K-means run: 4 false negatives with $K$ from 8 to 23, and (ii) Without quantization: same 4 false negatives with $T_{I}$ ranging from 0.08 to 1.2 .

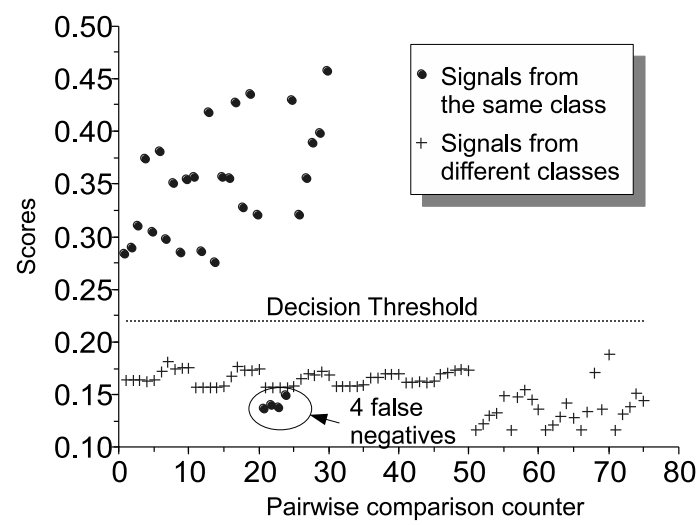

Figure 2: Comparison scores and classification of accelerometer signals - 30 cross-comparisons of signals from the same class, 75 cross-comparisons of signals from different classes, only 4 false negative results.

\section{CONCLUSION}

In this paper, the Lempel-Ziv complexity measure, usually associated to lossless compression of computer files, was used in a less usual task: pattern recognition. A modified version of the LZ analysis method, proposed in 1976 [1], was presented along with a new similarity measure aimed at comparing two sequences instead of computing complexity of a single one. This modified method, MLZ, uses the same simple but powerful ideas behind the original LZ method. Likewise, 
the new similarity measure uses the number of parsed segments instead of usual likelihood based measures used in pattern recognition.

Another contribution of this work was a method for continuously valued signal analysis through LZ algorithm. Thus, two strategies were studied, one based on signal space discretization in $K$ prototypes, through the K-Means algorithm, and another based on direct comparison between segments of multivariate signals in a continuous space.

To illustrate the usefulness of the proposed tools, two sets of experimental results were presented, namely: one on speaker identity verification (behavioural biometrics) and another on healthcare signal detection. All experiments yielded promising performances. As for experiments on biometrics with a single K-Means run, the EER of approximately $7.6 \%$ coincides with the one obtained through a classic pattern recognition method [16], for the same database and set of features. Besides, by using a strategy inspired in clustering ensembles, MLZ with and without quantization yielded almost the same result $(E E R \approx 5.2 \%)$. For the experiments with accelerometer signals (Healthcare) consistent detection results were obtained, with errors in only 4 out of 105 cross-comparisons for a wide range of algorithm parameters.

In both approaches, with and without quantization, either the number of prototypes, $K$, or the innovation threshold, $T_{I}$, were empirically tuned. Finding an optimum value for both $K$ and $T_{I}$, and studying the relationship between signal statistics and optimization is a matter for future research.

RESUMO. O conhecido método proposto por Abraham Lempel and Jacob Ziv para a análise de complexidade de sequências de símbolos foi modificado, neste trabalho, para comparar semelhanças entre suas sequências. Essa modificação permitiu a criação de um novo critério que pode substituir medidas de verossimilhança em algumas aplicações no campo do reconhecimento de padrões. Adicionalmente, no sentido de permitir a comparação de padrões multivariados em espaços contínuos, nós também apresentamos uma adaptação simples do método de Lempel-Ziv a sinais regularmente amostrados no tempo. Para ilustrar a utilidade das ferramentas propostas, dois conjuntos de resultados experimentais são apresentados e discutidos, a saber: um em verificação de locutor (biometria) e outro em detecção de sinais no monitoramento médico. Os dois experimentos produziram resultados promissores. Além disso, quando comparados a métodos tradicionais de reconhecimento de padrões, as novas abordagens produziram desempenhos superiores, em termos de taxas de erros iguais nos experimentos de identificação de orador.

Palavras-chave: análise de sinais, complexidade de Lempel-Ziv, modelagem sem a prioris.

\section{REFERENCES}

[1] A. Lempel \& J. Ziv. On the complexity of an individual sequences. IEEE Trans. on Inform. Theory, IT-22 (1976), 75-81. 
[2] C.E. Shannon. A mathematical theory of communication. Bell System Technical Journal, 27 (1948), $379-423$ and 623-656.

[3] T.M. Cover \& J.A. Thomas. Elements of Information Theory, John Wiley \& Sons, Inc. (2006).

[4] P. Johansen. Pattern recognition by data compression, in Proceedings of the 9th Scandinavian Conference on Image Analysis, (1995), 1131-1137.

[5] F. Kaspar \& H.G. Schuster. Easily calculable measure for the complexity of spatiotemporal patterns. Physical Review A, 36(2) (1987), 842-848.

[6] L.V. Batista \& M.M. Meira. Texture Classification Using the Lempel-Ziv-Welch Algorithm. In $C$ Science Advances in Artificial Intelligence - SBIA 2004, Lecture Notes in Computer Science, 3171, (2004), 444-453.

[7] J.M. Amigó, J. Szczepanski, E. Wajnryb \& M.V. Sanchez-Vives. Estimating the Entropy Rate of Spike Trains via Lempel-Ziv Compplexity. Neural Computation, 16 (2004), 717-736.

[8] L. Sarlabous, A. Torres, J.A. Fiz, J. Gea, J.B. Galdiz \& R. Jane. Multistate Lempel-Ziv (MLZ) index interpretation as a measure of amplitude and complexity changes. In Conf Proc IEEE Eng Med Biol Soc., (2009), 4375-4378.

[9] M. Aboy, R. Hornero, D. Abásolo \& D. Alvarez. Interpretation of the lempel-ziv complexity measure in the context of biomedical signal analysis. IEEE Trans. Biomed. Eng., 53(11) (2006), 2282-2288.

[10] M. Aboy, D. Cuesta-Frau, D. Austin \& P. Micó-Tormos. Characterization of Sample Entropy in the Context of Biomedical Signal Analysis. In Proceedings of the 29th Conf. Proc. IEEE Eng. Med. Biol. Soc., (2007), 5942-5945.

[11] Y. Gao, I. Kontoyiannis \& E. Bienenstock. Estimating the Entropy of Binary Time Series: Methodology, Some Theory and a Simulation Study. Entropy, 10 (2008), 71-99.

[12] P.E. Rapp, T.A.A. Watanabe, P. Faure \& C.J. Cellucci. Nonlinear Signal Classification. International Journal of Bifurcation and Chaos, 12(6) (2002), 1273-1293.

[13] J. Ziv \& A. Lempel. Compression of Individual Sequences via Variable-Rate Coding. IEEE Trans. on Inform. Theory, IT-24 (1978), 530-536.

[14] S. Theodoridis \& K. Koutroumbas. Pattern Recognition (2nd Ed.), Elsevier Academic Press (2003).

[15] A. Strehl \& J. Ghosh. Cluster Ensembles - A Knowledge Reuse Framework for Combining Multiple Partitions. Journal of Machine Learning Research, 3 (2002), 583-617.

[16] J. Montalvão \& M.R.R. Araujo. Is masking a relevant aspect lacking in MFCC? A speaker verification perspective. Pattern Recognition Letters, 33(16) (2012), 2156-2165.

[17] Blake, C.J. Merz. UCI repository of machine learning databases.

[18] B. Kaluza, V. Mirchevska, E. Dovgan, M. Lustrek \& M. Gams. An Agent-based Approach to Care in Independent Living, In Proceedings of the First international joint conference on Ambient intelligence, (2010), 177-186. 